Supporting information

for

\title{
Synthesis of Ether Oligomers
}

Olivier Renaudet and Jean-Louis Reymond*

Spectroscopic data of active compounds 25, 41, 49, 50, 75 and 76 and basic crystallographic data for oligomers 43 and $\mathbf{5 9}$. 
Compound 25: ${ }^{1} \mathrm{H}$ NMR (300 MHz, $\left.\mathrm{CDCl}_{3}\right): \delta=9.99(\mathrm{~s}, 1 \mathrm{H}), 7.51-7.24(\mathrm{~m}, 13 \mathrm{H})$, 7.09-7.03 (m, $8 \mathrm{H}), 6.97-6.94(\mathrm{~m}, 4 \mathrm{H}), 5.11(\mathrm{~s}, 2 \mathrm{H}), 5.09$ (s, $2 \mathrm{H}), 5.08(\mathrm{~s}, 2 \mathrm{H}), 5.07(\mathrm{~s}, 4 \mathrm{H}) ;{ }^{13} \mathrm{C} \mathrm{NMR}(75$ $\left.\mathrm{MHz}, \mathrm{CDCl}_{3}\right): \delta=192.1,159.2,159.0,159.0,138.5,138.5,137.9,137.8,136.9,130.1,129.8$, $129.7,128.6,128.0,127.5,123.7,122.2$, 120.0, 114.5, 114.4, 113.9, 113.9, 113.3, 70.0, 69.9, 69.8; HRESI-MS: calcd. for $\mathrm{C}_{42} \mathrm{H}_{36} \mathrm{O}_{6} \mathrm{Na}$ : 659.2409; found: 659.2413 [M+Na] ${ }^{+}$.

Compound 41: ${ }^{1} \mathrm{H}$ NMR (300 MHz, $\left.\mathrm{CDCl}_{3}\right): \delta=9.96(\mathrm{~s}, 1 \mathrm{H}), 8.38(\mathrm{~d}, 1 \mathrm{H}, J=2.1 \mathrm{~Hz}), 8.07$ (dd, $1 \mathrm{H}, J=8.7,2.1 \mathrm{~Hz}), 7.98(\mathrm{~d}, 1 \mathrm{H}, J=2.1 \mathrm{~Hz}), 7.60(\mathrm{~d}, 1 \mathrm{H}, J=8.7,2.3 \mathrm{~Hz}), 7.52-7.26(\mathrm{~m}, 8 \mathrm{H})$, $7.18(\mathrm{~d}, 1 \mathrm{H}, J=8.7 \mathrm{~Hz}), 6.88(\mathrm{~d}, 1 \mathrm{H}, J=9.0 \mathrm{~Hz}), 5.30(\mathrm{~s}, 2 \mathrm{H}), 5.28(\mathrm{~s}, 2 \mathrm{H}), 5.08$ (s, $2 \mathrm{H}), 2.32$ $(\mathrm{s}, 3 \mathrm{H}) ;{ }^{13} \mathrm{C}$ NMR $\left(75 \mathrm{MHz}\right.$, acetone- $\left.\mathrm{D}_{6}\right): \delta=194.6,161.9,160.9,156.2,141.4,139.7,138.2$, 135.8, 135.6, 134.6, 133.7, 133.3, 132.7, 132.6, 132.1, 132.0, 131.3, 129.2, 120.9, 120.7, 116.8, 76.6, 76.1, 73.3, 20.8; HREI-MS: calcd. for $\mathrm{C}_{29} \mathrm{H}_{24} \mathrm{~N}_{2} \mathrm{O}_{5}$ : 528.15326; found: $528.15375[\mathrm{M}+\mathrm{H}]^{+}$.

Compound 49: ${ }^{1} \mathrm{H}$ NMR (300 MHz, $\mathrm{CDCl}_{3}$ ): $\delta=10.42$ (s, $\left.1 \mathrm{H}\right), 8.17$ (d, $\left.1 \mathrm{H}, J=9.2 \mathrm{~Hz}\right), 8.09$ (d, $1 \mathrm{H}, J=9.0 \mathrm{~Hz}), 7.60(\mathrm{~d}, 1 \mathrm{H}, J=2.9 \mathrm{~Hz}), 7.36-7.30(\mathrm{~m}, 6 \mathrm{H}), 7.14(\mathrm{dd}, 1 \mathrm{H}, J=9.0,2.9 \mathrm{~Hz}), 6.92-$ $6.88(\mathrm{~m}, 4 \mathrm{H}), 5.50(\mathrm{~s}, 2 \mathrm{H}), 5.10(\mathrm{~s}, 2 \mathrm{H}), 5.07(\mathrm{~s}, 2 \mathrm{H}), 4.07(\mathrm{q}, 2 \mathrm{H}, J=7.0 \mathrm{~Hz}), 1.41(\mathrm{t}, 3 \mathrm{H}, J=$ $7.0 \mathrm{~Hz}) ;{ }^{13} \mathrm{C} \mathrm{NMR}\left(75 \mathrm{MHz}, \mathrm{CDCl}_{3}\right): \delta=188.5,163.4,163.0,160.6,149.4,148.2,139.6,137.7$, $135.5,134.3,128.8,128.6,128.5,127.8,127.6,127.3,120.6,119.4,114.6,114.2,114.1,113.4$, 112.8, 71.1, 70.6, 68.4, 64.5, 14.9; HREI-MS: calcd. for $\mathrm{C}_{30} \mathrm{H}_{26} \mathrm{~N}_{2} \mathrm{O}_{9}$ : 558.16383; found: 558.16431 $[\mathrm{M}+\mathrm{H}]^{+}$.

Compound 50: ${ }^{1} \mathrm{H}$ NMR $\left(300 \mathrm{MHz}, \mathrm{CDCl}_{3}\right): \delta=8.17(\mathrm{~d}, 1 \mathrm{H}, J=9.2 \mathrm{~Hz}), 8.11(\mathrm{~d}, 1 \mathrm{H}, J=9.0$ $\mathrm{Hz}), 7.61(\mathrm{~d}, 1 \mathrm{H}, J=2.8 \mathrm{~Hz}), 7.36-7.28(\mathrm{~m}, 5 \mathrm{H}), 7.25(\mathrm{~d}, 1 \mathrm{H}, J=2.8 \mathrm{~Hz}), 6.93-6.87(\mathrm{~m}, 5 \mathrm{H})$, $5.50(\mathrm{~s}, 2 \mathrm{H}), 5.11(\mathrm{~s}, 2 \mathrm{H}), 5.04(\mathrm{~s}, 2 \mathrm{H}), 4.92(\mathrm{~d}, 1 \mathrm{H}, J=6.1 \mathrm{~Hz}), 4.06(\mathrm{q}, 2 \mathrm{H}, J=7.0 \mathrm{~Hz}), 2.45$ (bt, $1 \mathrm{H}, J=6.1 \mathrm{~Hz}$ ), 1.41 (t, $3 \mathrm{H}, J=7.0 \mathrm{~Hz}$ ); HREI-MS: calcd. for $\mathrm{C}_{30} \mathrm{H}_{28} \mathrm{~N}_{2} \mathrm{O}_{9}$ : 560.17948; found: $560.17902[\mathrm{M}+\mathrm{H}]^{+}$.

Compound 75: ${ }^{1} \mathrm{H}$ NMR (300 MHz, $\left.\mathrm{CDCl}_{3}\right): \delta=10.50$ (s, $\left.1 \mathrm{H}\right), 8.16(\mathrm{~d}, 1 \mathrm{H}, J=9.0 \mathrm{~Hz}), 7.58$ (d, $1 \mathrm{H}, J=2.8 \mathrm{~Hz}), 7.45-7.38(\mathrm{~m}, 6 \mathrm{H}), 7.20(\mathrm{dd}, 1 \mathrm{H}, J=9.0,2.8 \mathrm{~Hz}), 7.17(\mathrm{dd}, 1 \mathrm{H}, J=8.7,2.6 \mathrm{~Hz})$, $6.97(\mathrm{~d}, 1 \mathrm{H}, J=1.9 \mathrm{~Hz}), 6.88(\mathrm{~d}, 1 \mathrm{H}, J=8.9 \mathrm{~Hz}), 6.84(\mathrm{dd}, 1 \mathrm{H}, J=8.1,2.1 \mathrm{~Hz}), 6.78(\mathrm{~d}, 1 \mathrm{H}, J=$ $8.3 \mathrm{~Hz}), 5.76(\mathrm{q}, 1 \mathrm{H}, J=6.4 \mathrm{~Hz}), 5.18(\mathrm{~d}, 1 \mathrm{H}, J=11.9 \mathrm{~Hz}), 5.11(\mathrm{~d}, 1 \mathrm{H}, J=11.9 \mathrm{~Hz}), 5.09$ (s, 2 $\mathrm{H}), 4.15(\mathrm{q}, 2 \mathrm{H}, J=7.0 \mathrm{~Hz}), 1.67(\mathrm{~d}, 1 \mathrm{H}, J=6.4 \mathrm{~Hz}), 1.51(\mathrm{~d}, 1 \mathrm{H}, J=7.0 \mathrm{~Hz}) ;{ }^{13} \mathrm{C} \mathrm{NMR}(75$ $\left.\mathrm{MHz}, \mathrm{CDCl}_{3}\right): \delta=188.5,163.1,153.4,149.7,148.2,142.3,136.6,134.3,133.8,128.7,128.2$, 
128.0, 127.8, 127.3, 127.2, 126.5, 126.4, 120.6, 119.3, 116.0, 114.2, 113.6, 113.0, 71.5, 71.2, 70.3, 64.8, 22.7, 14.9; HREI-MS: calcd. for $\mathrm{C}_{31} \mathrm{H}_{28} \mathrm{NO}_{7} \mathrm{Cl}$ : 561.15543; found: $561.15564[\mathrm{M}+\mathrm{H}]^{+}$.

Compound 76: ${ }^{1} \mathrm{H}$ NMR $\left(300 \mathrm{MHz}, \mathrm{CDCl}_{3}\right): \delta=8.19(\mathrm{~d}, 1 \mathrm{H}, J=9.0 \mathrm{~Hz}), 7.58(\mathrm{~d}, 1 \mathrm{H}, J=2.6$ Hz), 7.44-7.38 (m, 4 H), 7.32 (d, $1 \mathrm{H}, J=2.5 \mathrm{~Hz}), 7.17$ (dd, $1 \mathrm{H}, J=8.9,2.6 \mathrm{~Hz}), 6.97-6.93$ (m, 2 H), $6.88(\mathrm{~d}, 1 \mathrm{H}, J=8.7 \mathrm{~Hz}), 6.75(\mathrm{dd}, 2 \mathrm{H}, J=8.3,1.9 \mathrm{~Hz}), 6.77(\mathrm{~d}, 1 \mathrm{H}, J=8.4 \mathrm{~Hz}), 5.75(\mathrm{q}, 1 \mathrm{H}$, $J=6.3 \mathrm{~Hz}), 5.15(\mathrm{~d}, 1 \mathrm{H}, J=11.9 \mathrm{~Hz}), 5.10(\mathrm{~d}, 1 \mathrm{H}, J=11.9 \mathrm{~Hz}), 5.06(\mathrm{~s}, 2 \mathrm{H}), 5.01(\mathrm{~s}, 2 \mathrm{H}), 4.14$ $(\mathrm{q}, 2 \mathrm{H}, J=7.0 \mathrm{~Hz}), 1.66(\mathrm{~d}, 3 \mathrm{H}, J=6.3 \mathrm{~Hz}), 1.50(\mathrm{t}, 3 \mathrm{H}, J=7.0 \mathrm{~Hz})$; HREI-MS: calcd. for $\mathrm{C}_{31} \mathrm{H}_{30} \mathrm{NO}_{7} \mathrm{Cl}: 563.17108$; found: $563.17194[\mathrm{M}+\mathrm{H}]^{+}$. 


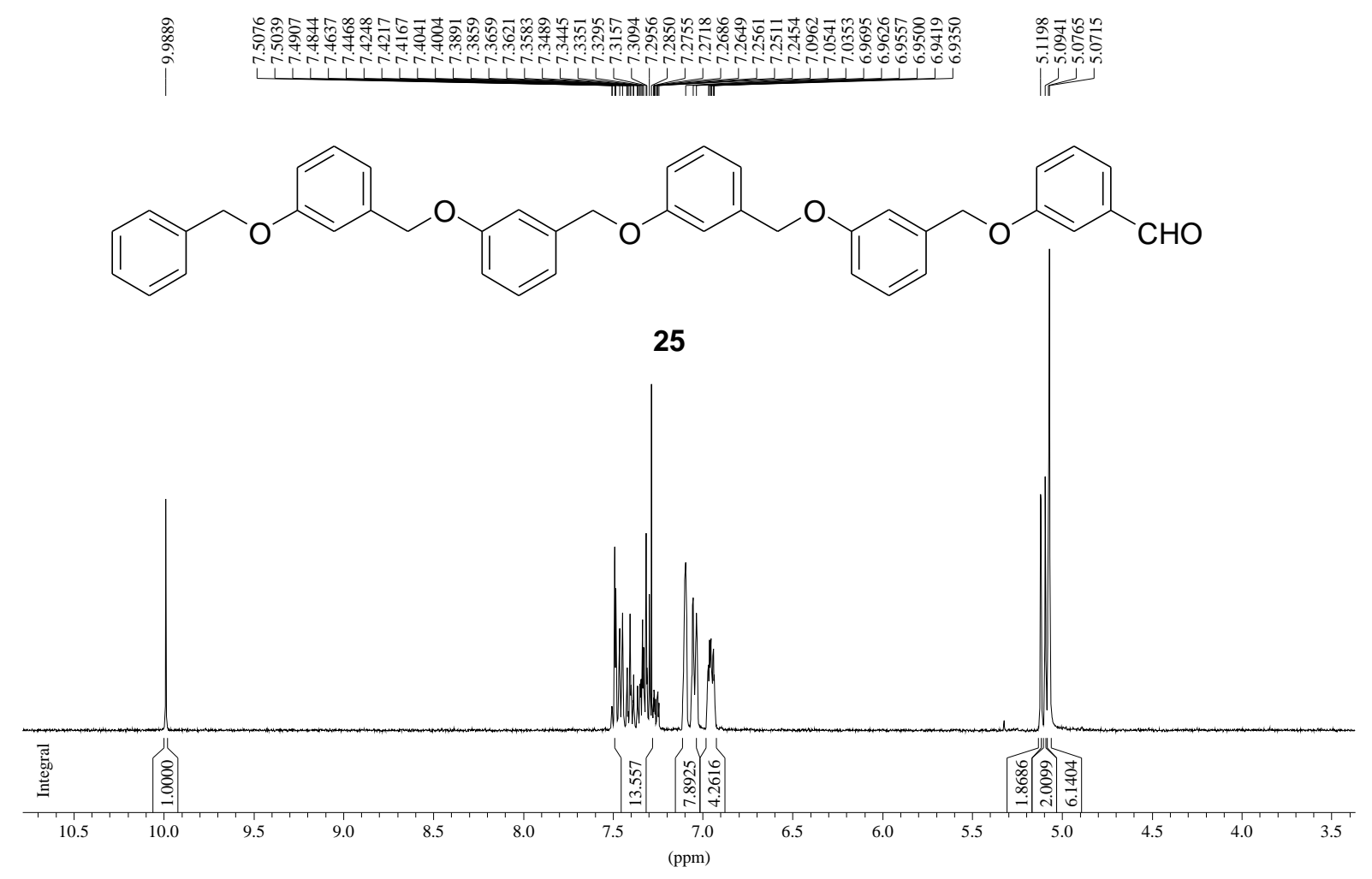

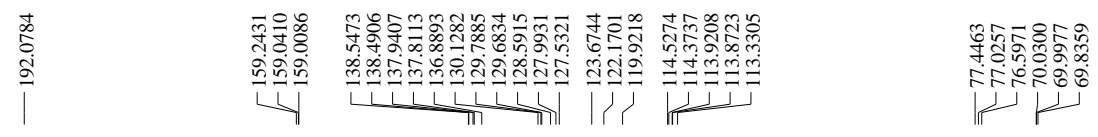

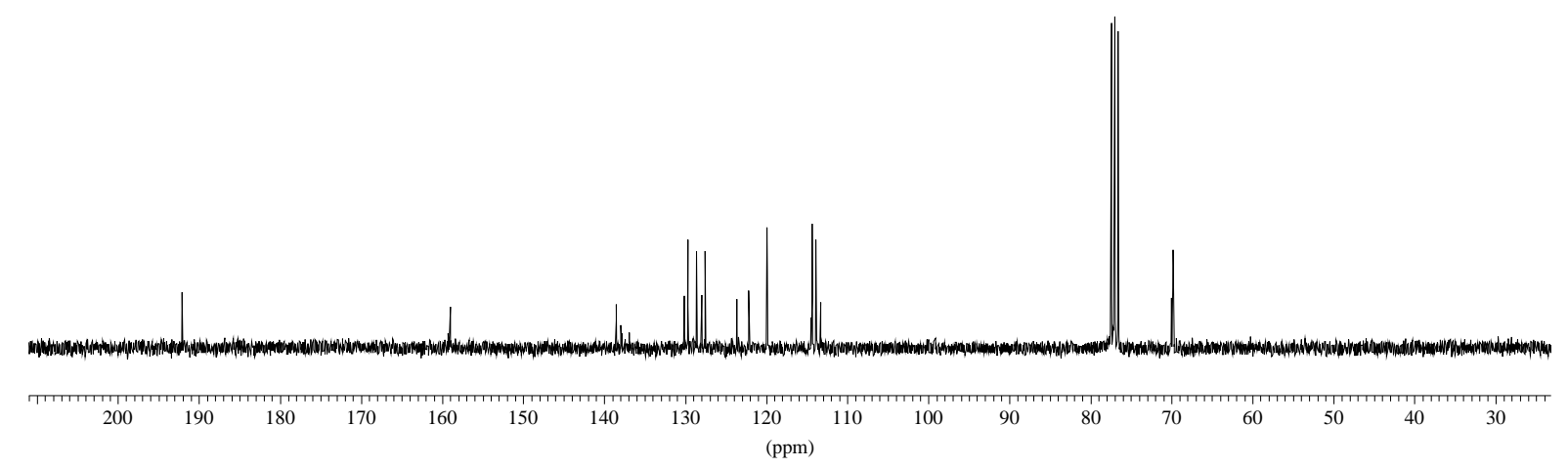



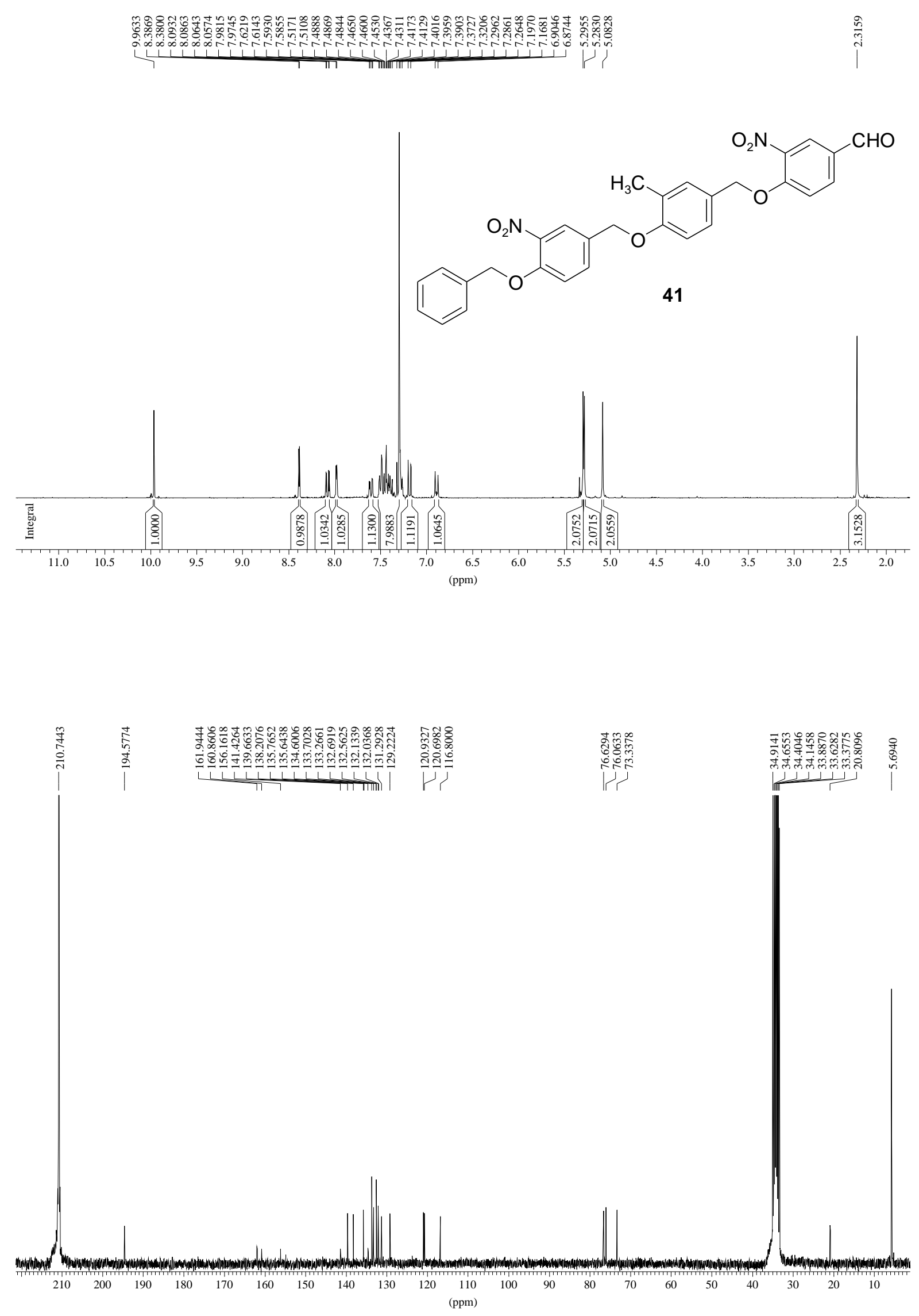

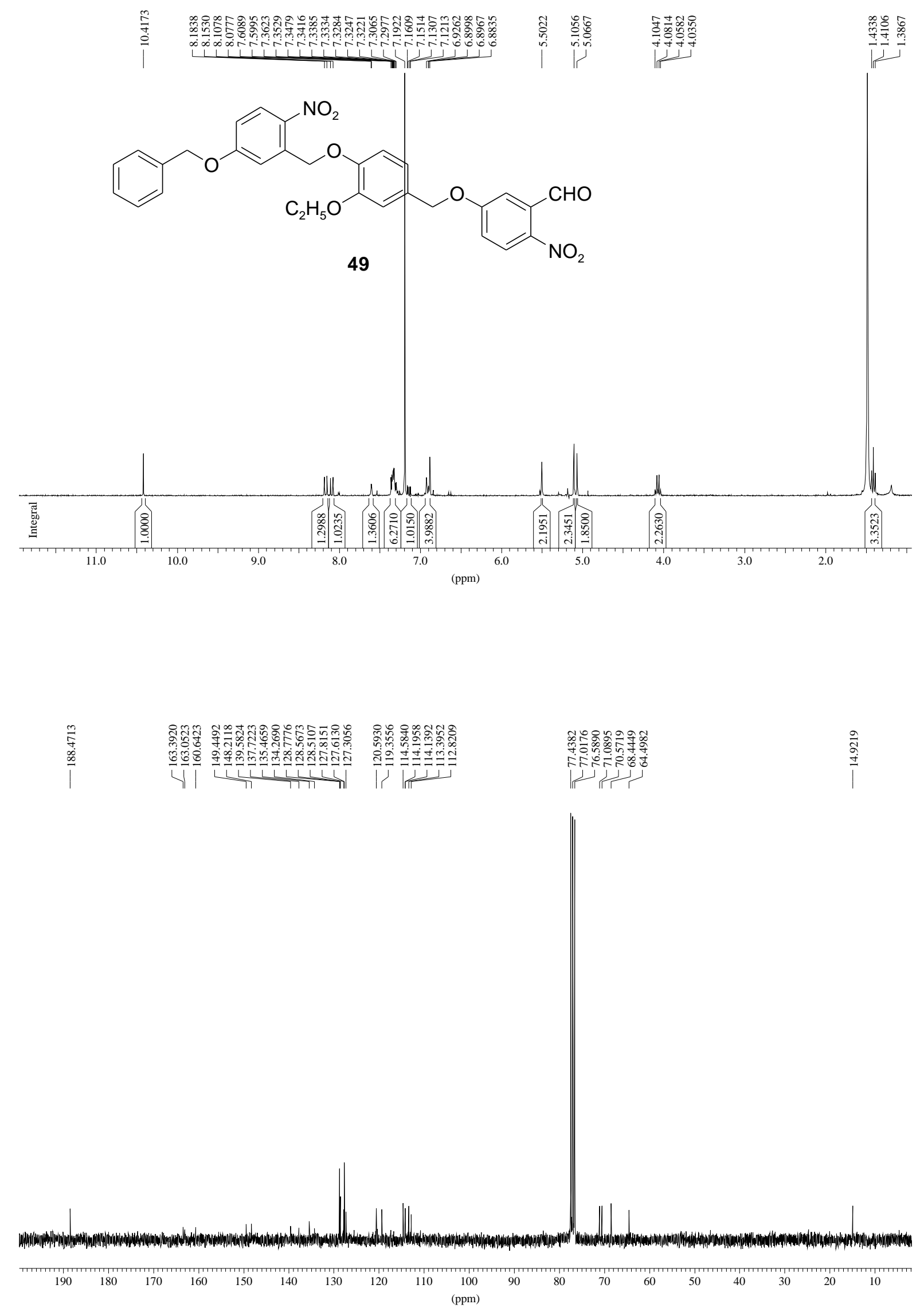

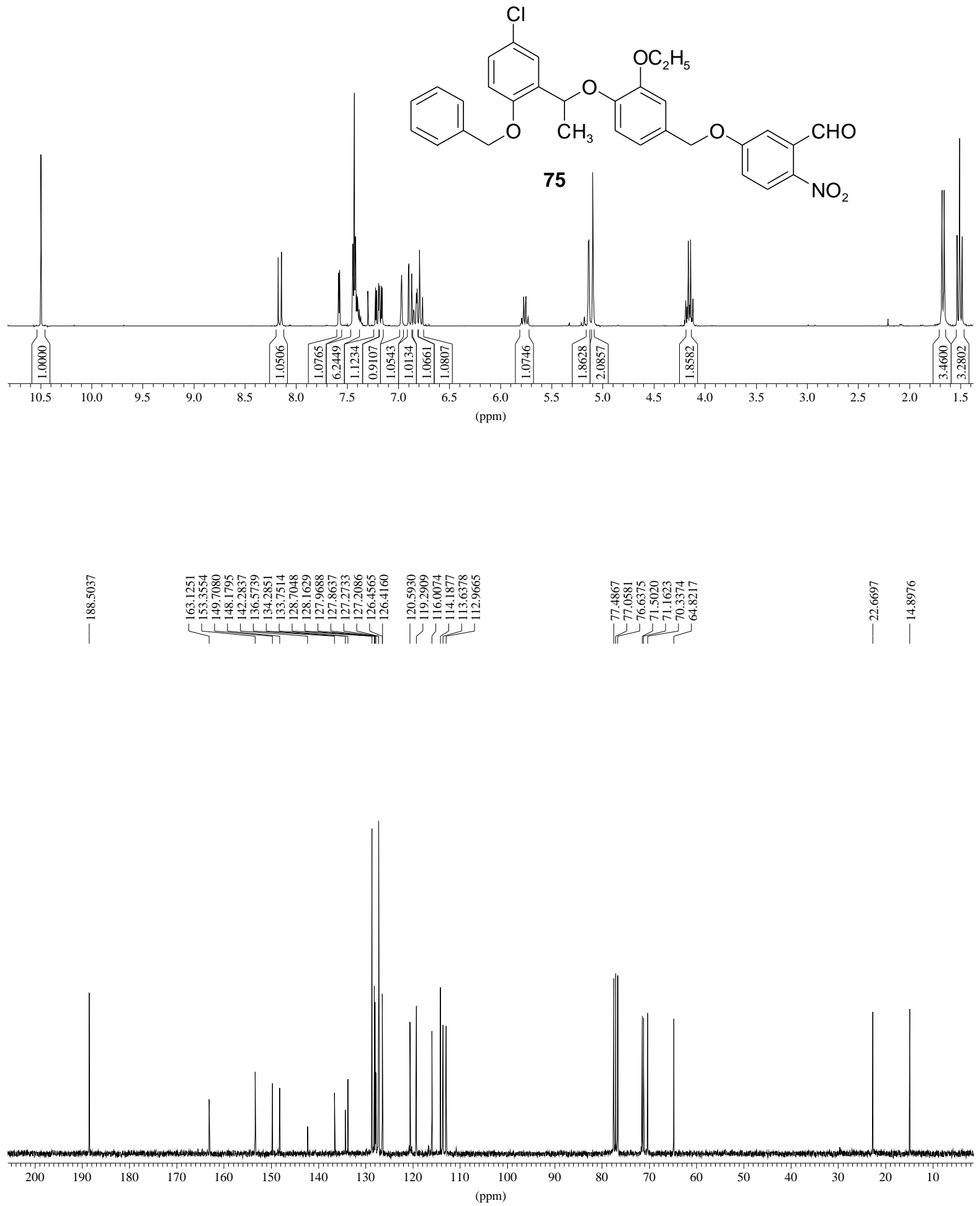
Crystal data table for $\mathbf{4 3}$.

\begin{tabular}{|c|c|}
\hline Crystal shape & plate \\
\hline Crystal colour & colourless \\
\hline Crystal size & $0.30 \times 0.30 \times 0.15 \mathrm{~mm}$ \\
\hline Empirical formula & C31 H29 N O7 \\
\hline Formula weight & 527.55 \\
\hline Crystal system & Triclinic \\
\hline Space group & $\mathrm{P}-1$ \\
\hline Unit cell dimensions & $\begin{array}{l}\mathrm{a}=9.5168(14) \mathrm{A} \quad \mathrm{al} \\
\mathrm{b}=10.0609(14) \mathrm{A} \\
\mathrm{c}=14.103(2) \mathrm{A} \quad \mathrm{gam}\end{array}$ \\
\hline Volume & $1272.2(3) \quad A^{\wedge} 3$ \\
\hline Cell refinement parameters & \\
\hline Reflections & 9865 \\
\hline Angle range & $1.99<$ theta $<26.04$ \\
\hline $\mathrm{z}$ & 2 \\
\hline Density (calculated) & $1.377 \mathrm{~g} / \mathrm{cm}^{\wedge} 3$ \\
\hline Radiation used & $\mathrm{MoK} \backslash \mathrm{a}$ \\
\hline Wavelength & $0.71073 \mathrm{~A}$ \\
\hline Linear absorption coefficient & $0.098 \mathrm{~mm}^{\wedge}-1$ \\
\hline Temperature & $153(2) \mathrm{K}$ \\
\hline
\end{tabular}


Crystal data table for 59.

Crystal shape

Crystal colour

Crystal size

Empirical formula

Formula weight

Crystal system

Space group

Unit cell dimensions

Volume

Cell refinement parameters

Reflections

Angle range

Density (calculated)

Radiation used

Wavelength

Linear absorption coefficient

Temperature plate

pale_yellow

$0.50 \times 0.50 \times 0.20 \mathrm{~mm}$

$\mathrm{C} 30 \mathrm{H} 27 \quad \mathrm{~N} \quad \mathrm{O} 8$

529.53

Triclinic

P -1

$\mathrm{a}=8.7426(8) \mathrm{A} \quad \mathrm{alpha}=96.122(7) \mathrm{deg}$

$\mathrm{b}=10.6484(9) \mathrm{A}$ beta $=99.555(7)$ deg.

$\mathrm{c}=14.4952(13) \mathrm{A}$ gamma $=104.529(7) \mathrm{deg}$.

$1272.5(2) A^{\wedge} 3$

16289

$1.93<$ theta $<29.59$

2

$1.382 \mathrm{~g} / \mathrm{cm}^{\wedge} 3$

$M \circ K \backslash a$

$0.71073 \mathrm{~A}$

$0.101 \mathrm{~mm}^{\wedge}-1$

153 (2) K 